\title{
Pareceristas do volume 36
}

Abigail Vital - Centro Federal de Educação Tecnológica Celso Suckow da Fonseca, Rio de Janeiro, RJ

Adriano José Ortiz - Instituto Federal do Paraná, Paranavaí, PR

Agostinho Serrano de Andrade Neto - Universidade Luterana do Brasil, Canoas, RS

Alan Alves Brito - Instituto de Física, Universidade Federal do Rio Grande do Sul, RS

Alberto Cupani - Departamento de Filosofia, UFSC, SC

Alcina M. T. Braz da Silva - Universidade Salgado de Oliveira, Niterói, RJ

Alessandra Riposati Arantes - Instituto de Física, Universidade Federal de Uberlândia, MG

Alessandro Damásio Trani Gomes - Departamento de Ciências Naturais, Universidade Federal de São João del-Rei, MG

Alexander Cunha - Instituto de Física, Universidade Federal do Rio Grande do Sul, Porto Alegre, RS

Alexandre C Tort - Instituto de Física, UFRJ

Alice Assis - Faculdade de Engenharia de Guaratinguetá, UNESP, Guaratingueta, SP

Alice Campos Pierson - Departamento de Metodologia de Ensino - UFSCar - SP

Aline Nicolli - Universidade Federal do Acre, Rio Branco, AC

Ana Paula Bispo da Silva - Centro de Ciências e Tecnologia, Universidade Estadual da Paraíba, PB

André Ary Leonel - Departamento de Metodologia de Ensino, Universidade Federal de Santa Catarina, SC

André Ferrer P. Martins - Departamento de Educação, Universidade Federal do Rio Grande do Norte, Natal, RN

André Koch Torres de Assis - Instituto de Física, UNICAMP, SP

André Coelho da Silva - Instituto Federal de Educação, Ciência e Tecnologia de São Paulo, Itapetininga, SP 
André Tenório Leite - Instituto Federal de Educação, Ciência e Tecnologia do Rio de Janeiro, Rio de Janeiro, RJ

Antônio Cesar do Prado Rosa Junior - Universidade Federal do Oeste da Bahia, Barreiras, BA

Ariane Baffa Lourenço - Grupo de Pesquisa em Ensino de Química de São Carlos, Universidade de São Paulo

Awdry Feisser Miquelin - Departamento Acadêmico de Física, Universidade Tecnológica Federal do Paraná, Curitiba, PR

Boniek Venceslau da Cruz Silva Universidade Federal do Piauí, Teresina, PI

Breno Arsioli Moura - Centro de Ciências Naturais e Humanas, Universidade Federal do ABC, Santo André, SP

Bruno dos Santos Simões - Universidade Federal da Grande Dourados, Dourados, MS

Caetano Roso - Programa de Pós-graduação em Educação Científica e Tecnológica, Universidade Federal de Santa Catarina, Florianópolis, SC

Caio Laganá - Instituto de Física Teórica, UNESP, SP

Camila Silveira da Silva - Universidade Federal do Paraná, Curitiba, PR

Carlos Aparecido Kantor - Faculdade de Filosofia Ciências e Letras, Centro Universitário Fundação Santo André, Santo André, SP

Cassiano Rezende Pagliarini - Universidade Federal de Ouro Preto, MG

Christina Helena Barboza - Museu de Astronomia e Ciências Afins, Rio de Janeiro, RJ

Cibelle Celestino Silva - Instituto de Física, Universidade de São Paulo, São Carlos, SP

Cláudia Adriana Silva - Universidade Federal do Tocantins, Palmas, TO

Cleci Werner da Rosa- Universidade de Passo Fundo, RS

Cristiano de Mattos - Universidade de São Paulo, Instituto de Física, São Paulo, SP

Cristiano Barbosa de Moura - Centro Federal de Educação Tecnológica Celso Suckow da Fonseca, Petrópolis, RJ

Daniel Gardelli - Departamento de Física, Universidade Estadual de Maringá, Maringá, PR

Daniel Juliano Pamplona da Silva - Universidade Federal de Alfenas, Poços de Caldas, MG

Danielle Nicolodelli - Universidade Federal da Fronteira Sul, Campus Realeza, PR 
Danilo Rodrigues César - Universidade Federal do Triângulo Mineiro, Uberaba, MG

Deise Miranda Vianna - Instituto de Física, Universidade Federal do Rio de Janeiro, RJ

Diego Alexandre Duarte - Universidade Federal de Santa Catarina, Joinville, SC

Diego Fabian Arevalo - Universidad Distrital Francisco José de Caldas, Bogotá, Colômbia

Diego Marceli Rocha - Universidade Federal de Campina Grande, Cajazeiras, PB

Dioni Pastorio - Instituto de Física, Universidade Federal do Rio Grande do Sul, Porto Alegre, RS

Dirceu da Silva - Departamento de Metodologia do Ensino, Universidade Estadual de Campinas, SP

Ederson Staudt - Universidade Federal do Amapá, Macapá, AP

Eduardo Kojy Takahashi - Instituto de Física, Universidade Federal de Uberlândia, MG

Elder Sales Teixeira - Departamento de Física, Universidade Estadual de Feira de Santana, BA

Eliane A. Veit - Instituto de Física, Universidade Federal do Rio Grande do Sul, Porto Alegre, RS

Elisabeth Barolli - Faculdade de Educação - UNICAMP - SP

Emerson Ferreira Gomes - Instituto Federal de Educação, Ciência e Tecnologia de São Paulo, Boituva, SP

Erick dos Santos Silva - Instituto Federal de Educação Ciência e Tecnologia de Mato Grosso, Juína, MT

Eugenio Maria de França Ramos - Universidade Estadual Paulista Júlio de Mesquita Filho, Rio Claro, SP

Fabiana Botelho Kneubil - Instituto Educacional Futuro da Ciência, São Paulo, SP

Fabio da Purificação de Bastos - Centro de Educação, Universidade Federal de Santa Maria, RS

Fábio Henrique de Alencar Freitas - Universidade Federal da Bahia, Salvador, BA

Fábio Marineli - Universidade Federal de Lavras, Lavras, MG 
Fábio Andrade de Moura - Instituto Federal de Educação, Ciência e Tecnologia do Pará, Bragança, PA

Fábio Bartolomeu Sanana - Departamento de Física, Universidade Federal de Santa Catarina, Florianópolis, SC

Fábio Saraiva da Rocha - Universidade Federal de Pelotas, RS

Fernanda Cátia Bozelli - Universidade Estadual Paulista Júlio de Mesquita Filho, Ilha Solteira, SP

Fernando Lang da Silveira - Instituto de Física, Universidade Federal do Rio Grande do Sul, Porto Alegre, RS

Flavia Polati Ferreira - Universidade Federal do Rio Grande do Norte, Natal, RN

Flávia Rezende - Laboratório de Tecnologias Cognitivas, NUTES, Universidade Federal do Rio de Janeiro, RJ

Frederico Ayres de Oliveira Neto - Universidade Federal de Mato Grosso, Cuiabá, MT

Gabriela Kaiana Ferreira - Universidade Federal de Santa Catarina, Florianópolis, SC

Geide Coelho - Universidade Federal do Espírito Santo, Vitoria, ES

Glauco dos Santos Ferreira da Silva - Centro Federal de Educação Tecnológica Celso Suckow

Graciella Watanabe - Universidade Federal do ABC, Santo André, SP

José Guilherme de Oliveira Brockington - Universidade Federal do ABC, Santo André, SP

Guilherme Stecca Marcom - E. E. Antônio Alves Aranha, Valinhos, SP

Hector Riveros - Instituto de Física, Universidad Nacional Autónoma de México, México

Henrique Cesar da Silva - Departamento de Metodologia do Ensino, Universidade Federal de Santa Catarina, Florianópolis, SC

Herica Cambraia Gomes - Centro Universitário de Volta Redonda, Volta Redonda, RJ

Indianara Lima Silva - Departamento de Física, Universidade Estadual de Feira de Santana, BA

Irlan von Lisingen - Departamento de Engenharia Mecânica, Universidade Federal de Santa Catarina, Florianopolis, SC

Ivã Gurgel - Faculdade de Educação, Universidade de São Paulo, SP 
Ivan Fortunato - Instituto Federal de São Paulo, Itapetininga, SP

Ivanderson Pereira da Silva - Universidade Federal de Alagoas, Arapiraca, AL

Ives Solano Araujo - Instituto de Física, UFRGS, RS

Ivoní de Freitas Reis - Universidade Federal de Juiz de Fora, Juiz de Fora, MG

Jader da Silva Neto - Instituto Federal de Educação, Ciência e Tecnologia do Rio Grande do Sul, Bento Gonçalves, RS

Jandrews Lins Gomes - Instituto Federal de Pernambuco, Campus Pesqueira, PE

João Alex Costa Carneiro - Centro Universitário Ítalo Brasileiro, São Paulo, SP

Jorge Cardoso Messeder - Instituto Federal de Educação, Ciência e Tecnologia do Rio de Janeiro, Arraial do Cabo, RJ

Jorge Cássio Costa Nóbriga - Universidade Federal de Santa Catarina, Blumenau, SC

José Alves da Silva - Universidade Federal de São Paulo, Campus Diadema, SP

José de Pinho Alves Neto - Instituto Federal de Educação, Ciência e Tecnologia de Santa Catarina, Florianópolis, SC

José Arthur Martins - Departamento de Física e Química, Universidade de Caxias do Sul, Caxias do Sul, RS

José Carlos de Jesus - Departamento de Física, Universidade Estadual de Feira de Santana, Feira de Santana, BA

José Cláudio Reis - Universidade do Estado do Rio de Janeiro, RJ

José de Pinho Alves Neto - Instituto Federal de Educação, Ciência e Tecnologia de Santa Catarina, SC

José Francisco Custódio Filho - Departamento de Física, Universidade Federal de Santa Catarina, $\mathrm{SC}$

José Otavio Baldinato - Instituto de Química, Universidade de São Paulo, São Paulo, SP

José Fernando Moura Rocha - Instituto de Física, Universidade Federal da Bahia, Salvador, BA

Josué Antunes de Macêdo - Instituto Federal de Educação, Ciência e Tecnologia do Norte de Minas Gerais, Montes Claros, MG 
Júlia Parreira - Pontifícia Universidade Católica de Minas Gerais, Belo Horizonte, MG

Juliana Machado - Centro Federal de Educação Tecnológica Celso Suckow da Fonseca, Rio de Janeiro, RJ

Juliana Mesquita Hidalgo - Departamento de Física Teórica e Experimental, Universidade Federal do Rio Grande do Norte, Natal, RN

Juliano Camillo - Departamento de Metodologia de Ensino, Universidade Federal de Santa Catarina, Florianópolis, SC

Karine Raquiel Halmenschlager - Universidade Federal de Santa Catarina, Florianópolis, SC

Katemari Diogo da Rosa - Instituto de Física, Universidade Federal da Bahia, BA

Kátia Cunha - Universidade Federal de Pernambuco, Campus do Agreste, Caruaru, PE

Laércio Ferracioli - Departamento de Física, UFES, ES

Laudileni Olenka - Universidade Federal de Rondônia, Porto Velho, RO

Leonardo Albuquerque Heidemann - Instituto de Física, Universidade Federal do Rio Grande do Sul, Porto Alegre, RS

Lúcia Helena Sasseron - Departamento de Metodologia do Ensino e Educação Comparada, Faculdade de Educação, Instituto de São Paulo, SP

Luciana Massi - Universidade Estadual Paulista "Julio Mesquita Filho", Campus de Araraquara, SP

Luciano Fernandes Silva - Instituto de Física e Química, Universidade Federal de Itajubá, Itajubá, MG

Luís Carlos Bassalo Crispino - Instituto de Ciências Exatas e Naturais, Universidade Federal do Pará, Belém, PA

Luiz Clement - Departamento de Física, Universidade do Estado de Santa Catarina, Joinville, SC

Luiz Henrique Arthury - Instituto Federal de Santa Catarina, Jaraguá do Sul

Luiz Marcelo Darroz - Neves Universidade de Passo Fundo, RS

Maína Bertagna Rocha - Instituto de Educação de Angra dos Reis, Angra dos Reis, RJ

Marcela Ribeiro da Silva - Secretaria de Estado de Educação de Minas Gerais, Belo Horizonte, $\mathrm{MG}$ 
Márcia Gorette Lima da Silva - Departamento de Química, Universidade Federal do Rio Grande do Norte, Natal, RN

Márcia Maria Lucchese - Universidade Federal do Pampa, Bagé, RS

Marcilia Barcellos - Centro Federal de Educação Tecnológica Celso Suckow da Fonseca, Petropolis, RJ

Marco Adriano Dias - Centro de Ciências e Educação Superior a Distância do Estado do Rio de Janeiro, RJ

Marcos Daniel Longhini - Faculdade de Educação, Universidade Federal de Uberlândia, MG

Marcos Fernandes Sobrinho - Instituto Federal Goiano, Campus Urutaí, GO

Maria Conceição Barbosa Lima - Departamento de Física, Universidade do Estado do Rio de Janeiro, RJ

Maria de Fatima Oliveira Saraiva - Instituto de Física, Universidade Federal do Rio Grande do Sul, Porto Alegre, RS

Maria Inês Affonseca Jardim - Universidade Anhnaguera, Campo Grande, MS

Maria Lúcia Netto Grillo - Instituto de Física, UERJ, RJ

Maria Madalena Dullius - UNIVATES, RS

Maria Rita Otero - Facultad de Ciencias Exactas, Universidad Nacional del Centro, Buenos Aires, Argentina

Marília Paixão Linhares - Universidade Estadual do Norte Fluminense, Rio de Janeiro, RJ

Marinez Meneghello Passos - Departamento de Matemática, Universidade Estadual de Londrina, PR

Marlon Alcântara - Instituto Federal do Sudeste de Minas Gerais, Juiz de Fora, MG

Marta de Almeida - Instituto de Filosofia e Ciências Humanas, Universidade do Estado do Rio de Janeiro, Rio de Janeiro, RJ

Matheus Monteiro Nascimento - Departamento de Física, Universidade Federal do Rio Grande do Sul, Porto Alegre, RS

Mauricio Girardi - Universidade Federal de Santa Catarina, Araranguá, SC

Mauricio Urban Kleinke - Instituto de Física, UNICAMP, SP 
Mikael Frank Rezende Junior - Departamento de Física e Química, Universidade Federal de Itajubá, MG

Nádia Cristina Guimarães Errobidart - Universidade Federal de Mato Grosso do Sul, Campo Grande, MS

Nadja Paraense - Instituto de Química, Universidade Federal do Rio de Janeiro, Rio de Janeiro, RJ

Nathan Lima - Instituto de Física, Universidade Federal do Rio Grande do Sul, Porto Alegre, RS

Nestor Cortez Saavedra Filho - Departamento Acadêmico de Física, Universidade Tecnológica Federal do Paraná, PR

Neusa Teresinha Massoni - Instituto de Física, Universidade Federal do Rio Grande do Sul, Porto Alegre, RS

Norberto Aranha - Universidade de Sorocaba, SP

Odilon Giovannini - Centro de Ciências Exatas e Tecnologia, Universidade de Caxias do Sul, RS

Orlando Gomes de Aguiar Junior - Departamento de Métodos e Técnicas de Ensino, Universidade Federal de Minas Gerais, Belo Horizonte, MG

Osmar Henrique Moura da Silva - Departamento de Física, Universidade Estadual de Londrina, PR

Otávio Bocheco - Instituto Federal Catarinense, Rio do Sul, SC

Paulo Henrique de Souza - Universidade Metropolitana de Santos, Santos, SP

Paulo José Sena dos Santos - Departamento de Física, Universidade Federal de Santa Catarina, SC

Paulo Maurício - Escola Superior de Educação de Lisboa, Lisboa, Portugal

Paulo Roberto Lima Jr. - Instituto de Física, Universidade de Brasília, Brasília, DF

Paulo V. dos S. Rebeque - Instituto Federal de Educação, Ciência e Tecnologia do Rio Grande do Sul, Campus Bento Gonçalves, RS

Pedro Donizete Colombo Junior - Instituto de Ciências Exatas, Universidade Federal do Triângulo Mineiro, Uberaba, MG

Pedro Fernando Teixeira Dorneles - Universidade Federal do Pampa, Bagé, RS 
Peter Alexander Bleinroth Schulz - Faculdade de Ciência Aplicadas, Universidade Estadual de Campinas, Limeira, SP

Rafael Alves Batista - Instituto de Astronomia, Universidade de São Paulo, São Paulo, SP

Roberto Soares da Cruz Hastenreiter - Instituto Federal de Educação, Ciência e Tecnologia do Rio de Janeiro, Niterói, RJ

Rodrigo Cuzinatto - Instituto de Ciência e Tecnologia, Universidade Federal de Alfenas, Poços de Caldas, MG

Rosemar Ayres dos Santos - Universidade Federal da Fronteira Sul, Cerro Largo, RS

Samir Lacerda da Silva - Instituto Federal do Espírito Santo, Coordenação de Física, Campus Vitória, ES

Sandro Livramento - Escola de Educação Básica Leonor de Barros, Florianópolis, SC

Sani Rutz da Silva - Universidade Tecnológica Federal do Paraná, Ponta Grossa, PR

Sebastião Rodrigues Moura - Instituto Federal de Educação, Ciência e Tecnologia do Pará, Parauapebas, PA

Sheila Cristina Ribeiro Rego - Centro Federal de Educação Tecnológica Celso Suckow da Fonseca, Rio de Janeiro, RJ

Silmar Antônio Travain - Universidade Estadual Paulista Júlio de Mesquita Filho, Guaratinguetá, SP

Silvania Souza do Nascimento - Faculdade de Educação, UFMG, MG

Simoni Tormöhlen Gehlen - Departamento de Ciências Exatas e Tecnológicas da Universidade Estadual de Santa Cruz, BA

Tania de Oliveira Camel - Escola Politécnica de Saúde Joaquim Venâncio (FIOCRUZ) e da Escola Técnica Estadual Ferreira Viana, Rio de Janeiro, RJ

Tânia Maria Garcia - Departamento de Teoria e Prática de Ensino, Universidade Federal do Paraná, Curitiba, PR

Thaís Cyrino de Mello Forato - Universidade Federal de São Paulo, Diadema, SP

Valéria Dias - Instituto de Física, Universidade de São Paulo, São Paulo, SP

Valéria Nunes Belmonte - Universidade Federal do Rio de Janeiro, Macae, RJ 
Vitor Luiz Bastos de Jesus - Instituto Federal de Educação, Ciência e Tecnologia do Rio de Janeiro, Nilópolis, RJ

Wagner Duarte José - Departamento de Ciências Exatas e Tecnológicas - Universidade de Santa Cruz - BA

Wagner Tadeu Jardim - Instituto Federal Sudeste de Minas Gerais, Juiz de Fora, MG

Wellington Pereira de Queirós - Universidade Federal do Mato Grosso do Sul, Pioneiros, MS

Wilmo Ernesto Francisco Junior - Universidade Federal de Alagoas, Campus Arapiraca, AL

Winston Gomes Schmiedecke - Instituto Federal de São Paulo, São Paulo, SP 